modèles

linguistiques

\section{Modèles linguistiques}

2| 2010

L'écriture mimétique : en hommage à Daniel Bilous

\title{
Anamorphoses mimétiques du temps proustien
}

Annexe

\section{Amandine Cyprès}

\section{(2) OpenEdition}

\section{Journals}

Édition électronique

URL : https://journals.openedition.org/ml/408

DOI : $10.4000 / \mathrm{ml} .408$

ISSN : 2274-0511

Éditeur

Association Modèles linguistiques

Édition imprimée

Date de publication : 1 novembre 2010

Pagination : 299-303

\section{Référence électronique}

Amandine Cyprès, "Anamorphoses mimétiques du temps proustien », Modèles linquistiques [En ligne],

2 | 2010, mis en ligne le 09 octobre 2013, consulté le 01 juillet 2021. URL : http://

journals.openedition.org/ml/408 ; DOI : https://doi.org/10.4000/ml.408

Ce document a été généré automatiquement le 1 juillet 2021.

(c) Modèles Linguistiques 


\title{
Anamorphoses mimétiques $\mathrm{du}$ temps proustien
}

Annexe

\author{
Amandine Cyprès
}

\section{Du côté de la Madeleine}

\author{
A la manière de M. P. \\ «Le seul véritable voyage, le seul bain de \\ Jouvence, ce ne serait pas d'aller vers de \\ nouveaux paysages, mais d'avoir d'autres yeux, \\ de voir l'univers avec les yeux d'un autre, de cent \\ autres, de voir les cent univers que chacun d'eux \\ voit, que chacun d'eux est » (Marcel Proust, La \\ Prisonnière).
}

1 Longtemps, je me suis promené de bonne heure. Parfois, à peine monté sur la plateforme arrière de l'omnibus, je n'avais pas eu le temps de dire «je m'assieds » et j'étais déjà bousculé. Or, soit que mon cerveau, informé par la brusque excitation de mes nerfs, n'eût pas classé le heurt dans la catégorie des sensations généralement tenues pour agréables, quoiqu'il pût paraitre tel à certaines personnes puisqu'il est vrai que les mêmes objets ne procurent pas toujours à tous les mêmes émotions, soit que cette information, même parvenue à mon cerveau, n'y eût pas rencontré l'écho qu'y aurait provoqué la résurrection du souvenir enfoui de quelque sensation ancienne, de même nature, perçue comme agréable en raison des circonstances ayant entouré son apparition, comme une cavalcade dans les couloirs du métropolitain ou une promenade en vaporetto sur le Grand Canal de Venise, ce genre d'accrochage ne me plaisait pas.

Il me rappelait les promenades matinales qui, bien des années auparavant, étaient l'objet de mes inquiétudes, depuis la veille qui les précédait, lorsque maman, montant me dire bonsoir dans ma petite chambre à la lanterne magique, me disait : «Demain, tu iras voir ta tante Léonie, ta visite lui fera plaisir, et tu t'assureras qu'elle n'a besoin de 
rien ; en revenant, tu ramèneras à Françoise des asperges du marché, qu'elle préparera à merveille " (en réalité, Françoise laissait à la fille de cuisine le soin d'éplucher les asperges). Si délicate que fût ma mère, elle ne pouvait se figurer à quel point ces promenades à la Madeleine, où habitait ma tante, m'inspirait la plus grande angoisse, en raison, non d'une aversion pour le style néoclassique et les colonnes corinthiennes de l'église de la Madeleine (qui ne le disputaient certes pas dans mon affection au style champêtre de l'église Saint-Hilaire de Combray, avec son vieux porche, ses dalles inégales, les beaux vitraux de son abside, et surtout la fine pointe de son clocher, auquel ma grand'mère reconnaissait, vertu suprême quoique non académique, un air «naturel» et "distingué »), ni d'un principe moral ou vœu mystique qui m'eût impérativement prescrit d'éviter ce quartier de Paris, ni même en raison des nombreux chocs que les transports en omnibus me laissaient présager, mais parce que cette visite à ma tante Léonie, en me faisant rentrer plus tard que d'habitude à la maison, aurait pour effet, si douloureux pour moi, de me faire partir au lit sitôt ma soupe prise, de sorte que ma mère retenue à table comme lorsque nous avions été nous promener le long de la propriété de Swann à Tansonville, du côté de Méséglise, ou comme lorsque du monde - c'est-à-dire Swann - venait dîner chez nous, ne monterait pas me dire bonsoir en m'embrassant. Pour l'apprenti écrivain et admirateur de Bergotte que j'étais, tout rempli des accents mélodiques et des flots harmonieux de poésie dont Bloch m'avait appris à me délecter " comme d'un breuvage nectaréen ", Swann n'était à cette époque pas encore auréolé du prestige insurpassable d'être l'ami du grand écrivain (ce que je devais découvrir plus tard), dont l'avis sur toutes sortes de sujets élevés, artistiques ou littéraires, lui était pain aussi quotidien que, pour moi, les considérations de ma tante Léonie sur le chien de Mme Sazerat, les étrennes d'Eulalie, sur le fait de savoir si Mme Goupil était arrivée à la messe avant l'élévation, ou ses commentaires sur l' « affaire », comme on disait alors parlant de l'affaire Lemoine - du nom de cet escroc qui avait failli réussir à convaincre le gouverneur de la De Beers d'acheter "le secret » de la fabrication des diamants -, et au sujet de laquelle je ne doutais pas que, avec son art consommé du contrepoint et des variations auquel j'eusse volontiers sacrifié n'importe lequel de mes essais littéraires, Bergotte eût trouvé, pour relater les péripéties et exalter la métaphore baudelairienne du charbon transformé en gemme, cent tournures plus variées, plus brillantes et plus précises que les bavardages de ma tante conférant sans fin avec Françoise, entre deux verres de pepsine à l'effet légèrement oblitéré par son état d'agitation, de «l'abaissement de la morale dans le monde de la finance " (fffinance, comme disait Françoise, en insistant sur la première syllabe) - monde qui incluait pour ma tante les diamantaires, mais dont elle avait la délicatesse d'exclure les agents de change, par égard pour Swann et son père, qu'avait bien connu mon grand-père -, ce que Françoise traduisait à sa façon, en constatant avec satisfaction (comme j'imaginais que le faisait la Pythie voyant se réaliser un de ses oracles) que «c'est toujours ceux qui ont les pépettes qui en veulent toujours plus, voilà d'où qu'ils piquent donc tant ", article tout droit sorti du code social portatif de notre cuisinière, de sa sagesse paysanne, populaire, immémoriale et universelle, qui avait réponse à tout, à tout ce qui, en tout cas, entrait dans son champ d'application, dans le cadre de sa "jurisprudence », ce qui lui permettait par exemple d'expliquer qu'on pût imiter la Crucifixion de Giotto à dessein d'abuser un collectionneur peu averti et d'alléger indûment son portefeuille, mais le rendait incurablement inapte à prétendre élucider les mobiles du faussaire de génie imitant le style d'un artiste à la perfection, non pour copier une œuvre existante et faire passer la copie pour l'original, mais pour 
enrichir le catalogue de l'artiste d'une œuvre nouvelle, imaginaire et originale, tel Hans Van Meegeren « inventant » avec ses pinceaux de faux Ver Meer de Delft, des Pèlerins d'Emmaüs, une Cène, ou encore un Christ et la femme adultère, lequel avait d'ailleurs occupé un temps une place de choix dans l'étude de Swann sur Ver Meer, avant qu'il ne s'aperçût de sa fausseté et ne parvînt à la conclusion que « cette femme adultère n'était pas son genre "; mais que Swann eût une autre vie que celle que nous lui connaissions, la vie d'un fils d'agent de change, et qu'il nous apparût tout différemment qu'aux autres personnes qu'il côtoyait, qu'il s'agît des membres du Jockey-Club ou de Mme de Villeparisis, fallait-il s'en étonner? tant il est vrai que les diverses facettes d'un individu ne nous apparaissent jamais simultanément et dépendent de l'observateur, de son milieu, de ses connaissances, de ses préjugés, de même que dépend de l'expérimentateur et de son "protocole » qu'il observera une onde ou un corpuscule, selon la mécanique ondulatoire du prince de Broglie - dont mon grand-père aimait à se faire raconter par Swann les petits faits de l'existence (du prince de Broglie, pas de la mécanique ondulatoire) - qui devait conduire à remettre en question le "réalisme » de la science, comme Bergotte remettait en cause dans sa belle philosophie le "réalisme " de l'art et son présupposé d'une réalité indépendante du point de vue du narrateur, comme si c'était le même Swann qui trônait dans les meilleurs salons du faubourg Saint-Germain et celui qui venait sonner le soir à la porte du jardin un panier de pêches à la main; et certes, j'aurais été bien étonné d'apprendre que cette église de la Madeleine devant laquelle je passais pour aller chez ma tante, Bergotte l'avait souvent visitée en compagnie de Gilberte Swann, cette demoiselle espiègle aux beaux cheveux roux, dont la rencontre m'avait fait voir son père, M. Swann, autrement que comme l'invité qui me privait par ses visites du dernier baiser de maman - drame de mon coucher dont je n'aurais voulu à aucun prix que son auteur inconscient le révélât à sa fille -, demoiselle à qui Bergotte servait de chaperon tout aussi naturellement que Françoise pour moi, quand, après avoir, comme elle disait, « aboutonné » mon paletot, elle m'accompagnait sur les Champs-Élysées, où je la traînais dès que la couleur du ciel et le baromètre de mon père me laissaient présager un temps propice aux jeux de Gilberte entre le guignol et le cirque, auxquels elle ne manquerait pas de m'associer quoique Mme Swann ne vit pas d'un bon œil tourner autour de sa fille «ce jeune homme avec un long cou comme un Greco, maigre comme un Carpaccio » - à moins que nous ne dissertassions de la brochure dans laquelle Bergotte parlait de Racine, citant ces vers

Quel funeste courroux emplit de sa fureur

Le flot de calomnies débordant votre cœur?

Quelle importune ardeur vous cause ce tourment?

Que me vaut cette haine sur ce char véhément?

vers que, contrairement à Bloch qui y voyait seulement la marque d'un zazou des plus subversifs et ne les trouvait « potables » qu'à la condition qu'ils ne signifiassent rien, je considérais comme des miroirs de la vérité que l'écrivain me tendait pour refléter dans mon esprit toutes les impressions que je ressentais afin de pouvoir les retranscrire un jour, dans cet omnibus à l'affluence active, sonore, odorante, colorée, pénétrante et surabondante, où j'étais bousculé de toutes parts, alors que nous remontions l'avenue du Bois, dans laquelle, depuis longtemps, ce n'étaient plus des calèches fraîchement blasonnées de la noblesse d'Empire qui croisaient et recroisaient l'attelage empanaché d'Odette Swann, mais des omnibus bondés doublés par des automobiles sans charme, dans lesquelles ne sont plus, hélas! Mme et Mlle Swann, qui ont fui comme les années - 
comme Albertine aussi, pour qui mon amour, comme le grand cru tourne au vinaigre, avait fini par tourner à la jalousie, avant de s'évaporer, lentement - et que je ne me rappelle plus aujourd'hui que grâce à la plaquette jaunie de Bergotte et son odeur un peu moisie : prestige de la littérature !

Un jour d'été, comme je rentrais chez moi accablé par la morne journée et la perspective d'une triste soirée, je sentis sur mon pied le contact d'une de ces chaussures qui semblent avoir été imitées des cothurnes antiques, portés par les glorieux ancêtres de la grande Berma. À l'instant même où la semelle mêlée de boue humide effleura mon escarpin verni, je tressaillis, attentif à ce qui se passait d'extraordinaire en moi. Un plaisir délicieux m'avait envahi et j'avais cessé de sentir les grondements, les ripostes, les querelles. D'où avait pu me venir cette puissante joie? Je sentais qu'elle était liée au contact de la chaussure bourbeuse, mais qu'elle le dépassait infiniment, ne devait pas être de la même nature. D'où venait-elle? Que signifiait-elle? Où l'appréhender? Je reçois une seconde poussée où je ne trouve rien de plus que dans la première, une troisième qui m'apporte un peu moins que la seconde. Il est temps que je l'arrête, la vertu de la bourrade semble diminuer. Que dira maman, en voyant l'état de mes effets si patiemment lavés, empesés, amidonnés par Françoise ? Je me pose sur une place libre et me tourne vers mon esprit. C'est à lui de trouver la vérité. Mais comment? Certes, ce qui palpite ainsi au fond de moi, ce doit être le signe, l'image, le souvenir visuel, qui, lié à ce piétinement, tente de le suivre jusqu'à moi. Mais arrivera-til jusqu'à la surface de ma claire conscience, ce souvenir, l'instant ancien que la traction d'un instant identique est venue de si loin solliciter, émouvoir, soulever tout au fond de mon être? Je ne sais. Est-il mort? Mort à jamais? Maintenant, je ne sens plus rien, il s'est arrêté, fatigué peut-être ; qui sait s'il remontera jamais dans le S ? La clochette de l'omnibus tinte en moi comme le grelot de la porte du jardin qui m'annonçait qu'enfin M. Swann était parti et que maman allait monter, première abdication de sa part à mes caprices et mon manque de volonté, dont elle marquait le début du déclin, ainsi que de celui de ma santé, et qui m'avait donné à la fois l'idée de mon œuvre et la crainte de ne pouvoir la réaliser.

Et tout d'un coup, deux heures plus tard, boulevard Malesherbes, dans le même omnibus mais dans l'autre sens, alors que je venais de croiser Bloch devant la gare Saint-Lazare (que je connaissais bien, avant même que la peinture de sa verrière par Monet ne m'en eût révélé l'essence passagère, ferrugineuse, fulminante, ferroviaire, pour y avoir pris jadis le train de Balbec, où j'avais fait la connaissance de Saint-Loup, dont le mariage avec Gilberte avait plus tard rapproché les côtés de Guermantes et de Méséglise, autant que le faisait l'omnibus pour la gare Saint-Lazare et cette place de la Madeleine si bien décrite par Bergotte, au dire de Swann - quoique Swann ne pût s'empêcher de le proclamer avec cette nuance d'ironie dans la voix qu'il adoptait toujours pour marquer qu'il ne voulait pas s'impliquer complètement dans l'opinion qu'il proférait) où il m'avait prodigué maint conseil vestimentaire utile, le souvenir m'était apparu. Ce coup, c'était celui qu'un lundi midi, jour où il y avait toujours grand monde sur la ligne $\mathrm{S}$, du côté de la Madeleine où j'allais voir tante Léonie, un individu entre le zist et le zest, hautain et bizarre, avec le même éclat dur dans le regard que le baron de Charlus - ce regard perçant qui m'avait tant frappé lorsque je l'avais croisé dans le raidillon d'aubépines du parc de Tansonville le jour même où j'avais rencontré Gilberte pour la première fois -, m'avait donné avant que je me jetasse sur un siège vide. 
6 La bousculade ne m'avait d'abord rien rappelé ; peut-être parce que, depuis longtemps, ma mémoire était comme cet omnibus, où se côtoyaient, serrés comme dans mon petit panier les poissons pêchés au bord de la Vivonne («serrés comme des harengs en caque!» disait Mme Verdurin), les Guermantes, les Cottard, les Brichot, les Verdurin, les Forcheville, qui m'apparaissaient soudain figés, "cloués » à leur siège, frappés de saisissement, comme ils l'eussent été à la vision terrible d'un implacable contrôleur venu leur demander leurs billets, dans cet omnibus où ils n'étaient plus autorisés depuis longtemps à voyager, face à cet éternel contrôleur qu'est le Temps.

Stéphane Tufféry : Le style, mode d'emploi, Cylibris/Littératures, 2002, p. 70-76 (avec l'aimable autorisation de l'auteur).

\section{AUTEUR}

AMANDINE CYPRÈS

Babel, EA 2649 